\title{
Inquiries into perinatal and early childhood deaths in a health care district
}

\author{
F BRIMBLECOMBE, M BASTOW, J JONES, N KENNEDY, AND J WADSWORTH \\ Paediatric Research Unit, Royal Devon and Exeter Hospital, Exeter
}

SUMMARY A controlled study was made of stillbirths and deaths of children under 2 years of age born to families residing within the boundaries of the Exeter District Health Authority between 1 October 1980 and 30 September 1981 . The study was concerned primarily with methodology and feasibility; the numbers involved were, with few exceptions, too small to permit significant conclusions to be drawn. In practice, the design and implementation of the project proved to be effective. It is suggested that if adopted as a routine service procedure in each health district, information not currently available may be retrieved. This information has epidemiological importance and will also help to identify local problems.

The research and development carried out by the Paediatric Research Unit at the Royal Devon and Exeter Hospital (Heavitree) ${ }^{1}$ has shown that the purposes identified by the Royal College of Obstetricians and Gynaecologists ${ }^{2}$ of perinatal audit and surveillance can be achieved. These may be summarised as follows:

(1) An improvement in the sharing of information by all health professionals providing perinatal and early childhood care resulting in beneficial continuing education and improvement in the quality of care provided.

(2) Data, which is not retrievable by existing methods can be collected in a non-attributable form. This data is valuable in the identification of local problems in a district health authority and is also suitable, if extended to other districts, for aggregation at regional and national level for the monitoring of factors which militate against further improvements in national perinatal and early childhood death rates.

(3) The procedure can be undertaken without breaching medical confidentiality.

\section{Methods}

It was planned that every perinatal death and death of a child under 2 years of age among families resident in the Exeter Health District during the survey year should be investigated. For each death a control family was selected for comparable investigation. The control family was matched for:

Residence by geographic area (city, urban, and rural).

Parity.

Date of conception.

Sex of children dying after the perinatal period. In the case of a 30 week stillbirth the details of the pregnancy for the matched control were identified up to the 30th week of pregnancy only, irrespective of the subsequent outcome. Similarly the details of the pregnancy and early life of a matched control for a child who died at 18 months of age were studied for the whole of that period. A steering committee consisting of key representatives from all the disciplines concerned with maternity and child health in the district was formed to advise and monitor the progress of the study.

Preparation. Before the pilot study began there was extensive local discussion with all the professional groups involved. It was considered essential for the successful implementation of the study that it should have the full support of all general practitioners, health visitors, community midwives, consultant obstetricians, hospital midwives, consultant paediatricians, pathologists, and community physicians. Undoubtedly, the final details of the methodology were improved greatly by these discussions. The local coroners also agreed to give their assistance and support. Every member of each 682 
before it began and was requested to inform the research team immediately a death occurred.

Collecting the information. As soon as the research team were informed of a death they telephoned the family's general practitioner asking him if he agreed to a full confidential enquiry, including a visit by a member of the research team to the bereaved parents. If the family doctor felt it inappropriate a modified enquiry took place restricted to information available from medical and nursing records. If the general practitioner agreed that a full investigation should take place, a letter was sent to the bereaved parents asking them if they were willing for a member of the research team to visit them. At the same time questionnaires were sent to the general practitioner, community midwife, and health visitor so that standardised information was obtained for each enquiry. Similarly, an approach was made to the consultant obstetrician and paediatrician, and the pathologist where appropriate. A necropsy protocol had been agreed with all the consultant pathologists (identical information was made available irrespective of whether the necropsy had been a routine procedure or was one ordered by the coroner).

All the home visits were made by one of the two research nurses, both of whom had extensive experience of children's nursing and community research. The visits were made approximately three weeks after the bereavement. The medical research fellow in the research team was responsible for collecting the medical information from the family doctor (and where appropriate clinical medical officer), consultant obstetrician, paediatrician, and pathologist. The two research nurses accepted a similar responsibility for the collection of information from the community and hospital midwives, health visitors, and where appropriate district nurses.

Case conference. Once all the information was available, including the report of the interview with the bereaved parents, a case conference was held. This was attended by:

(1) Service professionals directly concerned with the patient. General practitioner, community midwife, health visitor, consultant obstetrician, consultant paediatrician, and consultant pathologist.

(2) Research team. Research nurse, medical research fellow, Director of the research project (who acted as chairman of the conference).

(3) Members of the health district medical and nursing staff. Community physician, divisional nursing officer for community nursing services, divisional nursing officer for hospital midwifery service.

The case conference invariably started with an explanation of purpose by the chairman and an assurance that not only was the procedure confidential but that after the conclusion of the case conference any records subsequently retained would be in a non-attributable form with all traceable identification of the bereaved family and their professional attendants removed. The objective of the conference was set out under the following headings:

(1) To establish the cause of death (clinical and pathological diagnosis).

(2) To identify notable factors (medical and non-medical) in the pregnancy and early life of the child.

(3) To assess the standard of parental care.

(4) To assess the parents' use of services.

(5) To assess the parents' ease of access to community and hospital based services.

(6) To review the quality of communication between all the health agencies involved.

The information obtained about the control families was identical with regard to the home interview and questionnaires completed by all their professional attendants. It was not, however, considered justifiable to ask all their professional attendants to give time to attend a case conference. Thus the information available about them with regard to items 2 to 6 above was not strictly comparable with the information obtained about the fatal cases, but was collated by the research team from the home interview and the questionnaires completed by the families' professional attendants.

The plan of this study did not propose that an arbitrary decision should be made as to whether a particular death was 'avoidable' or 'inevitable'. It was the purpose to identify among both the index and control families 'notable' events, whether adverse or favourable, which may have influenced the course of the pregnancy and the early lives of the children. These notable events are divided into medical and 'non-medical' factors which, in the opinion of those who attended the case conferences, were considered sufficiently important to be included in the non-attributable records.

For all the stillbirths in which there was no evidence of congenital malformation we have made an arbitrary distinction between 'acute' and 'chronic' placental failure. By acute placental failure we simply mean that there was evidence that the fetus was alive at the time labour began. In contrast, where there was a history of absent 
fetal movements for at least 48 hours (and in most, cardiotachographic evidence of no fetal heart beat for at least 24 hours) before labour began, we have used the term chronic placental failure.

After the case conference the information from the home interview, data questionnaires, and case conference was transformed into coded information of a non-attributable kind and tabulated using a University of London computer. The original questionnaires were then destroyed as were all forms of linkage with individuals and their health records. The variety of notable factors concerning the index cases and the control group are illustrated in Table 1.

It is clear that no single source of health care notes (general practitioner, community midwife, health visitor, hospital obstetric, paediatric records, pathology records, or the death certificate) contained all the information that emerged at the case conferences. It is suggested that reviews of case records at a much later date would seldom provide so complete an account of all the circumstances than a home interview followed by a case conference attended by all those directly concerned and convened as soon as all the relevant information is available. The notable factors pertaining to the control group, although ascertained without a case conference, have provided a balance of information which can be set against the circumstances surrounding a perinatal or early childhood death. It is clear from Table 1 that in this small series there was no overall excess of notable factors among the index compared with the control cases. In many single instances there were multiple notable factors among both the index cases and their controls.

\section{Results}

The results are presented in the same sequence as the six stated purposes of the case conferences.

There were 62 deaths during the survey year comprising 30 stillbirths, 11 early neonatal deaths (age 0 to 6 days), four late neonatal deaths (age 7 to 28 days), and 17 deaths of children aged 1 month to 2 years of age. The full procedure (that is, completion of questionnaires, home interview, and case conference) was carried out for 51 of the 62 deaths. The full procedure was not carried out in the remaining 11 cases for the following reasons:

(1) Deaths to residents of the Exeter District Health Authority in which the stillbirth or neonatal

Table 1 Notable medical and medicosocial factors identified among perinatal deaths and matched controls

\begin{tabular}{|c|c|c|c|c|c|c|}
\hline \multirow[t]{2}{*}{ Notable factors } & \multicolumn{2}{|c|}{$\begin{array}{l}\text { Deaths associated } \\
\text { with congenital } \\
\text { malformations }\end{array}$} & \multicolumn{2}{|c|}{$\begin{array}{l}\text { Deaths associated } \\
\text { with chronic } \\
\text { placental failure }\end{array}$} & \multicolumn{2}{|c|}{$\begin{array}{l}\text { Deaths associated } \\
\text { with acute } \\
\text { placental failure }\end{array}$} \\
\hline & $\begin{array}{l}\text { Cases } \\
\text { (15) }\end{array}$ & $\begin{array}{l}\text { Controls } \\
\text { (14) }\end{array}$ & $\begin{array}{l}\text { Cases } \\
\text { (I7) }\end{array}$ & $\begin{array}{l}\text { Controls } \\
\text { (16) }\end{array}$ & $\begin{array}{l}\text { Cases } \\
\text { (9) }\end{array}$ & $\begin{array}{l}\text { Controls } \\
\text { (6) }\end{array}$ \\
\hline (1) Mother $<18$ years old & - & - & 1 & - & - & - \\
\hline (2) Mother $>37$ years old & 1 & - & 1 & 1 & - & - \\
\hline (3) Mother unmarried & & & & & & \\
\hline Cohabiting (longterm) & - & - & 1 & 1 & - & 1 \\
\hline Not regularly cohabiting. & 1 & 1 & 2 & 1 & - & - \\
\hline (4) Poor housing/finance & - & - & 1 & 1 & - & - \\
\hline (5) Smoked at least 10 cigarettes/day throughout pregnancy & - & 1 & 7 & 7 & 1 & - \\
\hline (6) High alcohol consumption throughout pregnancy & 1 & 2 & - & - & - & - \\
\hline (7) Maternal malnutrition & - & - & 1 & 1 & - & - \\
\hline $\begin{array}{l}\text { (8) Pre-existing maternal disease eg asthma, epilepsy, chronic pelvic } \\
\text { disease or inflammation }\end{array}$ & 1 & 2 & 1 & 1 & 2 & - \\
\hline (9) Maternal obesity & $i$ & 2 & 4 & - & 1 & 1 \\
\hline (10) History of subfertility & 3 & 3 & 1 & - & 2 & 1 \\
\hline (11) Previous termination of pregnancy & - & - & 1 & - & - & - \\
\hline (12) Undifferentiated febrile illness in first trimester & - & 1 & - & - & - & 1 \\
\hline (13) Stress in pregnancy & -- & 2 & 1 & - & 1 & - \\
\hline (14) Depression/attempted suicide & - & 1 & 2 & 4 & - & - \\
\hline (15) Accident in pregnancy causing injury or shock & - & 1 & - & 2 & - & - \\
\hline (16) Occupational hazard & 2 & - & 1 & - & - & 1 \\
\hline (17) Drugs in pregnancy & 3 & 2 & 1 & 3 & 1 & $\ldots$ \\
\hline (18) Irregular or incomplete antenatal attendance or lack of cooperation & - & - & 2 & 3 & - & - \\
\hline (19) Hyperemesis & - & - & 2 & 1 & - & - \\
\hline (20) Essential hypertension & - & 1 & 3 & - & - & - \\
\hline (21) Pre-cclampsia & 1 & 3 & 2 & 2 & - & 1 \\
\hline (22) Urinary infection (confirmed by laboratory) & - & 1 & 1 & - & - & - \\
\hline (23) Pronounced maternal weight loss in third trimester & - & - & - & 1 & - & 1 \\
\hline (24) Diminished fetal movements & 1 & 1 & 9 & 2 & 3 & - \\
\hline Total & 15 & 24 & 45 & 31 & 11 & 6 \\
\hline
\end{tabular}


death occurred in a hospital outside the district (4 cases).

(2) Consent withheld for two deaths which occurred in the first two days of the survey year; the two general practitioners involved were at that time insufficiently informed about the enquiry (2 cases).

(3) Delay of 6 months in information reaching the research team about a stillbirth (1 case).

(4) Compassionate grounds ( 2 cases).

(5) Litigation against the hospital was being considered by one family in which major postpartum maternal complications had occurred (1 case).

(6) Lack of understanding and cooperation from one family about the purpose of the enquiry (1 case).

Cause of death. After the cause of death had been established at the case conference a copy of the death certificate for each of the 41 perinatal deaths was requested from the Office of Population Censuses and Surveys (OPCS). It is notable that the case conference provided more information about the pathology, particularly in infants with congenital malformations, than was available from the death certificates. The case conference also provided much additional information about pathology relating to the placenta and its vessels. The OPCS inform us that during 1981 their normal procedures were in some respects disrupted by industrial action, thus the results which they kindly provided for us were in some respects atypical. Normally they would have been able to record more detailed information had they been able to make their standard enquiries where they considered that an initial death certificate did not provide them with all the information they normally received.

It is believed that the implementation of a confidential inquiry of this kind serves two additional purposes. Firstly, it stimulates doctors who sign initial death certificates to send on to the OPCS at a later date the additional information which only becomes available after the completion of the initial certificate. Secondly, information which is normally retrieved by health authorities from birth notification returns and nursing records may be both expanded and collated in a format which is of more practical use than is usual.

Necropsies. Necropsies, which followed the protocol previously agreed with the consultant pathologists were carried out in 52 of the 62 deaths in our survey. If the four deaths that occurred outside our own health district and the one stillbirth that was not brought to our attention until 6 months after the death had occurred are excluded, then it can be stated that necropsies following the agreed protocol were performed in 52 of the 57 deaths $(91 \%)$.

Wigglesworth classification. Wigglesworth suggested that the causes of perinatal deaths might usefully be classified into five groups. ${ }^{3}$

Normally formed macerated stillbirths.

Congential malformations.

Immaturity.

Asphyxial conditions in labour.

Other.

The British Births Survey of all infants born during one week of the month of March $1970^{4}$ included 410 perinatal deaths among the 17170 births during that week. We have reviewed the findings and classified them according to the Wigglesworth categories in Table 2 . In the present study, of 3185 births there were 41 perinatal deaths. These numbers are too small to achieve statistical significance but suggest a striking change in the profile of causation of perinatal deaths since 1970 .

Stillbirths. At the case conference (or by the research team alone in those instances where no case conference was held) a conclusion was reached about the cause of each of the 30 stillbirths. In 7 stillbirths a lethal congenital malformation was present, in 17 it was concluded that appreciable placental failure must have been present for more than two days before the start of labour, and in the remaining 6 either acute placental failure of less than 48 hours duration such as abruptio placenta or

Table 2 British Births Survey ${ }^{4}$ and results of present study classified according to Wigglesworth ${ }^{3}$

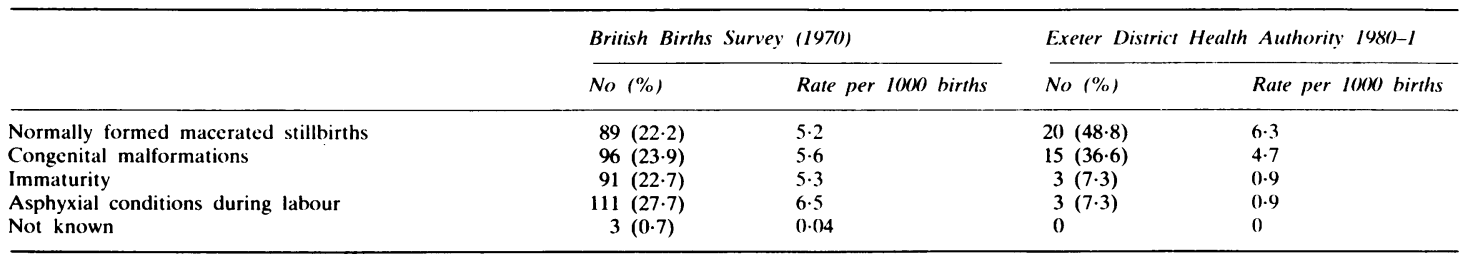


other intrapartum complications such as prolapsed cord had occurred.

Neonatal deaths. There were 15 neonatal deaths, 11 between 0 and 6 days and four between 7 and 28 days of life. These 15 neonatal deaths are considered together. In 11 instances a lethal malformation was the cause of death. There were three deaths associated with extreme prematurity $(25,26$, and 26 weeks' gestation). In two of these unsuccessful efforts had been made to suppress labour. If any of these three infants had been born dead they would have been recorded as abortions and would not have been included in this report, but because they were born alive they were recorded as neonatal deaths. This illustrates the inherent anomaly that currently exists in tables of perinatal rates as a result of the increasing number of survivors among infants born before 28 weeks' gestation. There were four late neonatal deaths ( 7 to 28 days). In three of these a lethal malformation was present; the remaining infant died of haemorrhagic disease of the newborn.

Deaths between 28 days and 2 years of life. There were 17 deaths among children aged between 1 month and 2 years. Five were the result of lethal congenital malformations, one an inborn error of metabolism, one acute infection, one an accident (inhalation of vomit), and 9 sudden infant death syndrome (SIDS). It is apparent in this small series that SIDS was the most common cause of death and that together with severe congenital malformations (including the one child with a severe inborn error of metabolism) accounted for all but two of the deaths between 1 month and 2 years of age.

Sudden infant death syndrome. Our study, apart from emphasising the high proportion of infant deaths due to SIDS, failed to shed any new light on this subject. Carpenter, Gardner, McWeeny, and Emery ${ }^{5}$ in Sheffield devised an 'At risk prediction score' designed to identify particular infants who were more likely to suffer SIDS. Studies in other areas have suggested that this scoring system, although relevant in Sheffield, was not applicable elsewhere. Only one of the 9 SIDS victims in our series and none of the matched controls showed a prediction score which would have indicated the risk of SIDS.

Four of the 9 mothers of SIDS victims were recorded as having suffered either notable stress or a chronic anxiety state during pregnancy, or received conflicting advice about child care. Three of their 9 matched control mothers had suffered notable maternal stress and one had been greatly distressed by the experience of having labour induced.
Additional results. In addition to the results reported in this paper, the study showed much about the social characteristics of the study and control populations, their use of and access to services, including the state of pregnancy at which professional advice was sought, the standard of parental care, communication between patients and professionals and interprofessional communication. Much insight was gained into the needs (met and unmet) of families with regard to bereavement. In all these areas practical information was obtained which would otherwise have been undocumented. These results are reported in detail elsewhere. ${ }^{1}$

Confidentiality. Before the start of the study it was determined by all concerned that this was to be a confidential scientific enquiry. All the questionnaires were separately filed and seen only by the medical and nursing members of the research team and by a trusted medical secretary. From the questionnaires the data was transferred into nonattributable code numbers as was all the information from the home interviews and case conferences. Once the latter had taken place all attributable information was destroyed. All that remains of an attributable nature is the usual medical and nursing records that are normally retained by any medical and nursing service. During the course of the analysis the code numbers were again changed and the original code numbers were also destroyed. No one attended the case conferences apart from the doctors and nurses directly concerned with the patient, the community physician, the divisional nursing officers of community and hospital midwifery services, the medical and nursing members of the research team, and the medical secretary.

All the parents were aware that a scientific study was being undertaken which it was hoped might lead to a reduction in perinatal and early childhood mortality in the future. At the case conference a decision was taken, determined by their general practitioner, as to their need for further counselling as a result of the investigation. $\mathrm{He}$ also decided whether he would undertake this responsibility himself or delegate it to a colleague in a different discipline (midwife, health visitor, hospital consultant, or geneticist).

The system proved workable and effective in practice. No examples of breach of confidentiality have been reported to the research team as a result of the study.

\section{Conclusions}

Although the usual conventional title is given to this section of the report, a more modest word which 
would convey better the authors' meaning would be 'suggestions'

(1) The first and principal suggestion is that a confidential enquiry into every perinatal and early childhood death would be a valuable service procedure in every health district.

(2) The information in this report illustrates the range of data of a local nature which can be retrieved by this procedure including:

(i) Demographic changes.

(ii) Distribution of health service provision for maternity and child health within the health district and their relation to patient needs.

(iii) Improved identification of the causes of perinatal and early childhood deaths as a continuing process sensitive to secular changes.

(iv) Identification of notable medical and medicosocial factors which are currently relevant to early mortality.

(v) A continuing assessment of standards of parental care and parents' use of and access to services.

(vi) A continuing audit of the quality of communication between families and health service professionals and interprofessional communication.

(3) Information retrieved in this way in each health district would provide more useful epidemiological data than that currently available at regional or national level.

(4) It is not for the authors to attempt to provide more than an indication of the resources required. It is our belief that the following staff would be sufficient to sustain a continuing enquiry:

(i) The designation of two senior doctors as alternates (consultant obstetrician, paediatrician, pathologist, district medical officer, or general practitioner) as chairmen of the district inquiry who would be required to give one session per week to this work. (ii) The appointment of two part time nurses (health visitor and midwife) who would each be employed for half of their working week.

(iii) Appropriate secretarial assistance.

(5) The essential prerequisities of the inquiry are: (i) The cooperation of all health professionals including attendance at the case conference concerning their patient.

(i) A standard necropsy protocol is recommended.

(iii) A home interview of the bereaved parents by the designated nurses (see 4(ii) above).

(iv) A case conference at which the appropriate non-attributable data is identified.

(v) The data processing of this information into a usable form.

(vi) The meticulous preservation of the confidentiality of the inquiry process.

(6) We suggest that not only would a continuing process of audit of this kind help identify priority needs for improving maternity and child services, it would also serve as a valuable method of continuing inservice education for all health professionals.

\section{References}

1 Brimblecombe FSW, Bastow M, Jones J, Kennedy N, Wadsworth J. A suggested model for inquiries into perinatal and early childhood deaths in a health care district. Liverpool: Children's Research Fund, 1983.

2 Perinatal audit and surveillance. London: Proceedings of the eighth study group of the Royal College of Obstetricians and Gynaecologists, 1980.

3 Wigglesworth JS. Monitoring perinatal mortality. A pathological approach. Lancet 1980;ii:684.

4 Chamberlain R, Chamberlain G, Howlett B. Claireaux A. British Births 1970. Vol 1. London: Heinemann Medical Books, 1975.

5 Carpenter RG, Gardner A, McWeeny PM, Emery JL. Multistage scoring system for identifying infants at risk of unexpected death. Arch Dis Child 1977;52:606.

Correspondence to Professor F S W Brimblecombe, Royal Devon and Exeter Hospital (Heavitree), Gladstone Road, Exeter EX1 2ED. 